\title{
Percent Change From Baseline
}

National Cancer Institute

\section{Source}

National Cancer Institute. Percent Change From Baseline. NCI Thesaurus. Code 696621.

The value of the last measurement taken divided by the initial value taken at some baseline event or timepoint. 